Lucía Gutiérrez*, Sonia Romero, Gustavo B. da Silva, Rocío Costo, Maria D. Vargas, Célia M. Ronconi, Carlos J. Serna, Sabino Veintemillas-Verdaguer and María del Puerto Morales*

\title{
Degradation of magnetic nanoparticles mimicking lysosomal conditions followed by AC susceptibility
}

DOI 10.1515/bmt-2015-0043

Received March 5, 2015; accepted May 7, 2015; online first June 2, 2015

\section{Abstract}

Background: A deeper knowledge on the effects of the degradation of magnetic nanoparticles on their magnetic properties is required to develop tools for the identification and quantification of magnetic nanoparticles in biological media by magnetic means.

Methods: Citric acid and phosphonoacetic acid-coated magnetic nanoparticles have been degraded in a medium that mimics lysosomal conditions. Magnetic measurements and transmission electron microscopy have been used to follow up the degradation process.

Results: Particle size is reduced significantly in $24 \mathrm{~h}$ at $\mathrm{pH}$ 4.5 and body temperature. These transformations affect the magnetic properties of the compounds. A reduction of the interparticle interactions is observed just $4 \mathrm{~h}$ after the beginning of the degradation process. A strong paramagnetic contribution coming from the degradation products appears with time.

*Corresponding authors: Lucía Gutiérrez and María del Puerto Morales, Instituto de Ciencia de Materiales de Madrid (ICMM)/CSIC, Department of Biomaterials and Bioinspired Materials, Sor Juana Inés de la Cruz 3, Cantoblanco, 28049 Madrid, Spain, Phone: +3491334 9000, E-mail: lucia@icmm.csic.es, puerto@icmm.csic.es. http://orcid.org/0000-0003-2366-3598 (LG) Sonia Romero, Rocío Costo, Carlos J. Serna and Sabino Veintemillas-Verdaguer: Instituto de Ciencia de Materiales de Madrid (ICMM)/CSIC, Department of Biomaterials and Bioinspired Materials, Sor Juana Inés de la Cruz 3, Cantoblanco, 28049 Madrid, Spain

Gustavo B. da Silva: Instituto de Ciencia de Materiales de Madrid (ICMM)/CSIC, Department of Biomaterials and Bioinspired

Materials, Sor Juana Inés de la Cruz 3, Cantoblanco, 28049 Madrid, Spain; and Instituto de Química, Universidade Federal Fluminense, Campus do Valonguinho, Centro, 24020-150, Niterói, RJ, Brazil

Maria D. Vargas and Célia M. Ronconi: Instituto de Química, Universidade Federal Fluminense, Campus do Valonguinho, Centro, 24020-150, Niterói, RJ, Brazil
Conclusions: A model for the in vivo degradation of magnetic nanoparticles has been followed to gain insight on the changes of the magnetic properties of iron oxides during their degradation. The degradation kinetics is affected by the particle coating, in our case being the phosphonoacetic acid-coated particles degraded faster than the citric acid-coated ones.

Keywords: biomedical applications; iron oxides; maghemite; magnetic characterization.

\section{Background}

The number of biomedical applications for magnetic nanoparticles has grown steadily in the last few years [8]. In addition to the most widely developed applications, such as contrast agents for magnetic resonance imaging (MRI) [31], heating mediators for magnetic hyperthermia in cancer treatment [18], and drug delivery systems [11], novel applications include the use of magnetic particles for wound healing [3, 26], stroke treatment [2], tissue engineering [29], and parasite diagnosis [5]. In this scenario, it is necessary to specifically produce compounds with structural characteristics suitable for each application [14].

A few remaining challenges need to be addressed before the use of nanoparticles is widely accepted in biomedical applications. First, detailed knowledge of the particle toxicity is required before the material reaches the clinical practice. The toxic potential of the particles has been thoroughly analyzed in cell cultures [4]; however, $3 \mathrm{D}$ cell cultures may behave in a different way to their 2D counterparts [20]. Moreover, although the particle toxicity could be low, little information is usually collected on the toxicity of the particle degradation products.

When the analysis shifts from cells to animals, a number of issues arise. It is still not clear how to control the particle biodistribution once they have been administered. Novel approaches include testing the advantages 
of using external magnetic fields [15], special antibodies [30], or specific particle coatings [7] to selectively target the area of interest to treat a disease or diagnose it. In addition, the fate of the particles after systemic injection has not been properly characterized, i.e., the residence time of the particles in tissues and the metabolic routes activated during the particle degradation need to be assessed.

Only a few studies on the biodistribution and degradation of magnetic nanoparticles in animal models have been reported, mainly due to the difficulties in analyzing the particles in tissue matrices, particularly as the animal size increases. Most of the biomedical applications require small amounts of material to achieve the desired effect; therefore, in animal experiments, the particles end up being extremely diluted within tissue matrices, hindering the evaluation of their degradation processes. One of the biggest problems faced in the characterization of magnetic nanoparticles in tissues arises from the fact that most particles used for biomedical applications are based on iron oxides, and there is a large amount of endogenous iron already in the tissues. Thus, the distinction between the iron from the particles and endogenous iron present in the tissue is a complicated task [6]. Techniques that exploit the specific magnetic properties of the particles and, therefore, discern them from tissue iron-containing species are currently being used to analyze magnetic nanoparticles in tissue samples, in particular electron spin resonance (ESR) [6], ferromagnetic resonance [12], field-dependent magnetization [28], or alternating current (AC) magnetic susceptibility [16] measurements. In the latter case, the temperature dependence of the AC magnetic susceptibility has been proven to be a powerful tool for the quantitative analysis of magnetic nanoparticles and biogenic species in animal tissue samples, as it allows the study of the particle biodistribution and degradation processes [27]. Of special interest is the fact that both diamagnetic and paramagnetic contributions to the out-of-phase susceptibility are zero, allowing the facile determination of the presence of magnetic nanoparticles and/or biomineral iron (e.g., ferritin, the iron storage protein) [17]. However, there are still a few remaining issues which will need to be solved before this technique can be adopted as a gold standard for the study of the magnetic nanoparticle fate. When studying the presence of magnetic nanoparticles in tissue samples during their degradation, the temperature position of the out-of phase susceptibility maxima is mainly affected by two parameters, the interparticle interactions and the particle size reduction due to their degradation. Both the disaggregation of the particles reducing the interparticle interactions and the degradation of the particles reducing their size, shift the temperature position of the out-of-phase susceptibility maxima toward lower temperatures [17]. Therefore, the identification of which of the two processes is being observed is not obvious when a shift on the temperature location is observed with time.

The aim of our work is to shed some light on the evolution of the magnetic properties of iron oxide nanoparticles during a degradation process that mimics in vivo conditions. In particular, we focus on the effect of the particle degradation on the temperature dependence of the $\mathrm{AC}$ magnetic susceptibility of the particles, as this technique has been revealed as a powerful technique for quantitative analysis of magnetic nanoparticles and biogenic species in animal tissue samples. The development of standards that mimic the degradation processes in tissues is fundamental to quantify the presence of nanoparticles in tissues and follow its long-term transformation.

\section{Methods}

\section{Magnetic nanoparticle synthesis}

Iron oxide magnetic nanoparticles (MNPs) were synthesized in aqueous media, following a modified Massart method [25]. Briefly, $75 \mathrm{ml}$ of a $\mathrm{NH}_{4} \mathrm{OH}$ solution ( $25 \%$, from Fluka - Riedel de Haën, Germany) was rapidly added to a solution of $\mathrm{FeCl}_{2}$ ( $0.175 \mathrm{M}$, from Sigma Aldrich, Germany, $\geq 99.0 \%$ ) and $\mathrm{FeCl}_{3}$ (0.334 $\mathrm{M}$, from VWR International, France, $27 \%$ Aqueous solution). The addition was carried out at room temperature under vigorous stirring. After $5 \mathrm{~min}$, the sample was washed three times with distilled water by magnetic decantation. Particles were then treated with nitric acid and iron nitrate to fully oxidize the magnetic iron oxide to maghemite, activate their surface, and improve the colloidal properties [9]. In addition, maghemite $\left(\mathrm{Fe}_{3} \mathrm{O}_{4}\right)$ is a more stable iron oxide phase, and up to now, the only one approved by the FDA (U.S. Food and Drug Administration) for in vivo administration. Briefly, the particles were treated with $300 \mathrm{ml}$ of $2 \mathrm{M}$ aqueous solution of $\mathrm{HNO}_{3}$ (from Sigma Aldrich, Germany, $\geq 65 \%$ ) and stirred for $15 \mathrm{~min}$. Then, the supernatant was removed, and $75 \mathrm{ml}$ of aqueous solution of $\mathrm{Fe}\left(\mathrm{NO}_{3}\right)_{3}(1 \mathrm{M}$, from Sigma Aldrich, Spain, $\geq 98 \%$ ) and $130 \mathrm{ml}$ of distilled water were added, followed by refluxing for $30 \mathrm{~min}$. After cooling down and removing the supernatant, $300 \mathrm{ml}$ of $2 \mathrm{M}$ aqueous solution of $\mathrm{HNO}_{3}$ was added, and the sample was stirred for $15 \mathrm{~min}$. The sample was washed three times with acetone and redispersed in distilled water. The acetone residue was rota-evaporated and the resulting product named MNPs.

Particles (MNPs) were subsequently coated with two different anionic compounds, citric acid (from Sigma Aldrich, Austria, $\geq 99.5 \%$ ) and phosphonoacetic acid (from Sigma Aldrich, Canada, 98\%). We used a standard procedure to generate the citric acid-coated magnetic nanoparticles (CIT-MNPs) [21, 24]. Briefly, $40 \mathrm{ml}$ of a solution of $0.1 \mathrm{M}$ citric acid was added dropwise to $50 \mathrm{ml}$ of treated sample ([Fe] $\approx 20 \mathrm{mg} /$ $\mathrm{ml})$ at $\mathrm{pH} 3$ before being heated at $80^{\circ} \mathrm{C}$ for 30 min under vigorous mechanical stirring.

Phosphonoacetic acid-coated magnetic nanoparticles (PAAMNPs) were prepared following a protocol that has been previously 
described [10]. Briefly, $10 \mathrm{ml}$ of acid-treated sample ([Fe] $77 \mathrm{mg} / \mathrm{ml})$ was dispersed in $50 \mathrm{ml}$ of distilled water at $\mathrm{pH} 2.5$. Then, $40 \mathrm{ml}$ of a solution of $0.05 \mathrm{M}$ phosphonoacetic acid was added dropwise under sonication. Samples were sonicated in a bath during $30 \mathrm{~min}$, after which time the dispersion became turbid due to the coating of the nanoparticles.

For both samples, the excess of the coating was washed by centrifugation and dialyzed in 51 of distilled water by using a membrane with a 12,000-14,000 nominal molecular weight cut off. The $\mathrm{pH}$ of the dispersions was adjusted first to 11 with $1 \mathrm{~m} \mathrm{KOH} \mathrm{(from} \mathrm{Sigma}$ Aldrich, Czech Republic, $\geq 85 \%$ ) and then to 7 with $1 \mathrm{M} \mathrm{HNO}_{3}$, and PBS buffer (from Sigma Aldrich, USA) was added in the same conditions that would be used for in vivo applications.

\section{Degradation of magnetic nanoparticles mimicking lysosomal conditions}

The effect of the $\mathrm{pH}$ on the kinetics of the degradation process was evaluated at room temperature during 15 days using citrate buffer solutions at different concentrations of citric acid $(20 \mathrm{mmol} / \mathrm{l}$, from Sigma-Aldrich, Austria, >99.5\%) and different pHs (2.5, 3.5, and 4.5), conditions that have been previously used by Lévy et al. [22]. Different volumes of the synthetized particles were added to the citrate buffer solutions to reach a final iron concentration of $1 \mathrm{mmol} / \mathrm{l}$. A second experiment was prepared, using higher temperatures $\left(37^{\circ} \mathrm{C}\right)$, to simulate body temperature, and $\mathrm{pH} 4.5$, to mimic lysosomal conditions, similar to the protocol described by Arbab et al. [1]. The degradation medium with the particles was split into several tubes and placed in a hot block. The degradation was stopped at $0 \mathrm{~h}, 4 \mathrm{~h}$, and $24 \mathrm{~h}$ by either freezing the samples at $-20^{\circ} \mathrm{C}$ or drying the sample for the transmission electron microscopy (TEM) observation. The hydrodynamic size measurements that require liquid samples were performed immediately at these time points.

\section{Magnetic nanoparticle characterization}

Iron determination was carried out in an inductively coupled plasmaoptical emission spectrometry (ICP-OES) apparatus, OPTIME 2100DV from Perkin Elmer (USA) after acid digestion.

Structural characterization: The crystal structure of the as-synthetized nanoparticles was identified by X-ray powder diffraction performed in a Bruker (USA) D8 Advance powder diffractometer by using the $\mathrm{CuK} \alpha$ radiation with an energy-discriminator (Sol-X) detector. Infrared spectra of the original samples diluted in $2 \% \mathrm{KBr}$ were recorded between 4000 and $250 \mathrm{~cm}^{-1}$ in a Bruker (USA) IFS 66V-S spectrometer and a Nicolet FT-IR 20SXC (Thermo Scientific, USA). Thermal analysis was performed to quantify the amount of coating molecules on the particle surface. Simultaneous thermogravimetric (TG) and differential thermal analysis (DTA) were performed on a Seiko TG/DTA 320U thermobalance (Seiko Instruments, Japan). Samples were heated from room temperature to $900^{\circ} \mathrm{C}$ at $10^{\circ} \mathrm{C} / \mathrm{min}$ under an air flow of $100 \mathrm{ml} / \mathrm{min}$.

Size analysis: A Zetasizer nano ZS by Malvern Instruments (UK) was used to determine both the hydrodynamic size and Z-potential.

To minimize the effect of multiple scattering in the hydrodynamic size distribution analysis, measurements at three different sample concentrations were performed. The result with the higher count number and the best position was selected as correct. It is worthy to mention that although the fundamental size distribution generated by dynamic light scattering (DLS) measurements is an intensity distribution, this can be converted to a volume distribution using the refraction index (2.42 for maghemite) and the Mie theory. This volume distribution can be further converted to a number distribution; however, number distributions are of limited use as small errors in gathering data could lead to huge errors in distribution by number. In most of the experiments of this work, the hydrodynamic size distribution is measured in terms of volume instead of intensity. When we measure in intensity mode, we are overestimating the contribution of the large particles. This is because large particles scatter much more light than small particles (the intensity of scattering of a particle is proportional to the sixth power of its diameter - from Rayleigh's approximation).

Particle core size was determined from TEM micrographs using a JEOL JEM1010 (100 KV) microscope (Japan). For observation of the sample in the microscope, particles were dispersed in isopropanol or water, and a drop of the suspension was placed onto a copper grid covered by a carbon film and allowed to dry at room temperature. Mean particle size and distribution were evaluated by measuring the largest internal dimension of at least 150 particles.

Magnetic characterization: For the magnetic characterization of the degradation products, liquid samples were frozen once the desired degradation time was reached and freeze dried overnight in a LyoQuest freeze dryer (Telstar, Spain). The resulting solid sample was compacted into gelatin capsules for magnetic characterization. This sample preparation procedure prevents particle movement through Brown rotation in an alternating field. Hysteresis loops with a maximum field of $5 \mathrm{~T}$ were measured in a Vibrating Sample Magnetometer (MLVSM9, MagLab 9T, Oxford Instruments, UK). AC magnetic susceptibility measurements were performed in a Quantum Design (USA) MPMS-XL SQUID magnetometer with magnetic field amplitude of $0.41 \mathrm{mT}$ and a frequency of $11 \mathrm{~Hz}$ in the temperature range between 2 and $300 \mathrm{~K}$.

\section{Results}

\section{As-synthetized magnetic nanoparticle characterization}

Maghemite nanoparticles with nearly spherical morphology and an average size of around $6.5 \mathrm{~nm}$, measured from TEM images, were obtained by the coprecipitation method and subsequent acid treatment. The X-ray diffraction spectra from the magnetic nanoparticles were indexed to an inverse spinel structure (JCPDS 39-1346) (Figure 1). The average crystallite size was calculated by Scherrer's formula using the half width of the (311) X-ray diffraction peak to be $6 \mathrm{~nm}$, in agreement with the TEM observations. The average particle size of the coated particles was $6.5 \pm 1.7 \mathrm{~nm}$ and $6.8 \pm 1.8 \mathrm{~nm}$ for the citric acid and phosphonoacetic acid coatings, respectively (Figure 2). No 

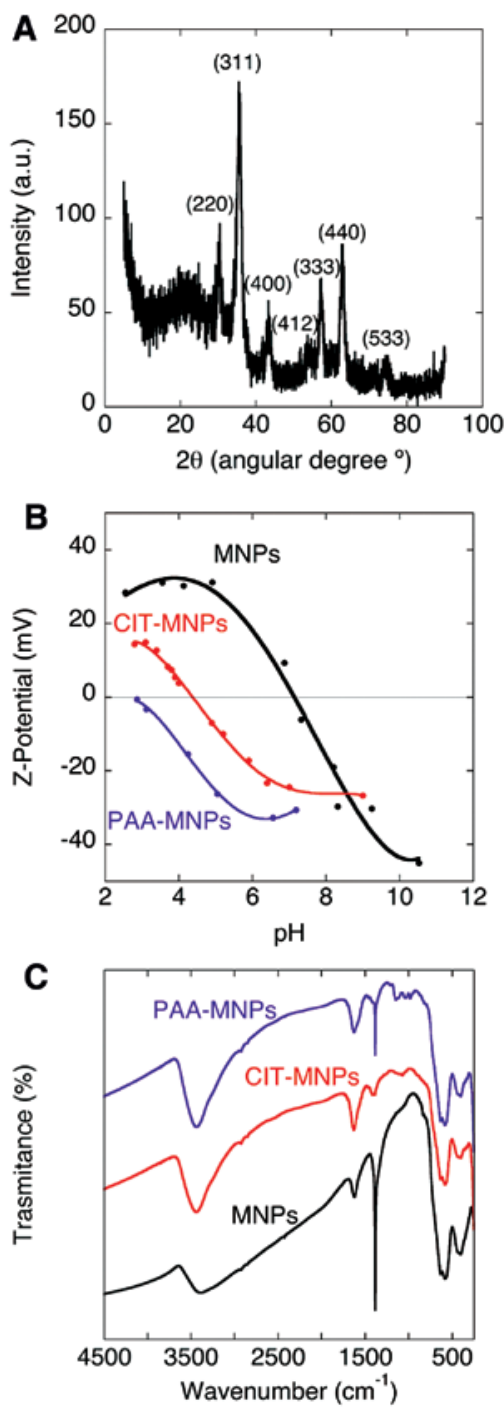

Figure 1: Particle's characterization.

(A) XRD pattern of the sample MNPs prior to the coating procedure, (B) Z-Potential versus $\mathrm{pH}$, and (C) FTIR spectra of the uncoated maghemite nanoparticles and the citric acid and phosphonoacetic acid-coated nanoparticles.

significant size modification was observed after the particle coating, as both samples were prepared from the same batch of magnetic cores.

The FTIR spectra of the maghemite nanoparticles before and after the coatings are shown in Figure 1. The typical bands of maghemite are centered at $640,575,440$, and $400 \mathrm{~cm}^{-1}$. A sharp band at $1381 \mathrm{~cm}^{-1}$ is due to the presence of nitric acid, which is used during the acid treatment to oxidize the particles and to adjust the $\mathrm{pH}$ of the samples. Bands at 1630 and $3440 \mathrm{~cm}^{-1}$ are typical of water chemi- or physi-sorbed onto the particle surface. The presence of the coating molecules onto the particle surface is evidenced by the bands between 1250 and $800 \mathrm{~cm}^{-1}$ : In the phosphonoacetic acid-coated nanoparticles, the presence
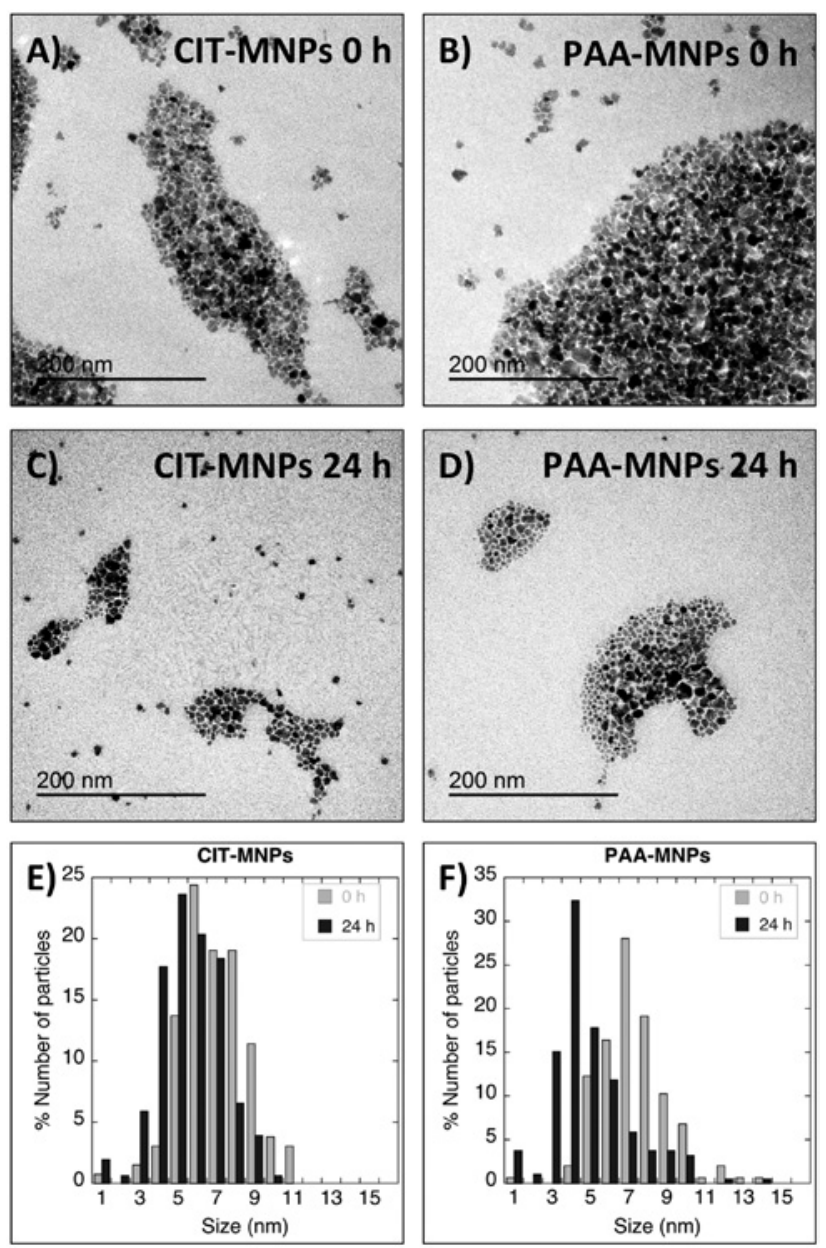

Figure 2: Size reduction during the degradation process mimicking lysosomal conditions $\left(37^{\circ} \mathrm{C}, \mathrm{pH}=4.5\right)$.

Transmission electron micrographs of the particles at the initial ( $A$ and $B$ ) and final points ( $C$ and $D$ ) of the degradation process together with the particle core size distribution measurements obtained from the TEM images (E and F).

of adsorption band at 980 and around $1208 \mathrm{~cm}^{-1}$ would be characteristic of $\mathrm{POH}$ and $\mathrm{P}=\mathrm{O}$ stretching bands, respectively, while the P-O stretching bands are forming a broad band at $1036 \mathrm{~cm}^{-1}$. On the other hand, samples coated with citric acid present bands at $1070 \mathrm{~cm}^{-1}$, which correspond to the $\rho-\left(\mathrm{CH}_{3}\right)$ vibrations, while bands at 1112 and $1260 \mathrm{~cm}^{-1}$ are attributable to $\mathrm{v}-(\mathrm{C}-\mathrm{OH})$ and the carboxylic group, respectively.

Consequently, a shift in the isoelectric point was observed from $\mathrm{pH} 7$ (MNPs) to 4.4 and 2.8 for the particles coated with citric acid and phosphonoacetic acid, respectively. Thus, at $\mathrm{pH}$, coated particles were electrostatically stabilized on the light of the large Z-potential values: -26 and $-32 \mathrm{mV}$ for samples PAA-MNPs and CITMNPs, respectively. As a result of these large surface charges, particles were colloidally stable, and the average 
hydrodynamic size of both samples was below $100 \mathrm{~nm}$ at the initial time point of the degradation process.

Thermal analysis revealed a total weight loss of around $10 \%$ (10.23\% and $9.7 \%$ for CIT-MNPs and PAAMNPs, respectively). In both cases, the majority of the weight loss takes place before $400^{\circ} \mathrm{C}$ due to the organic content of the coating molecules.

\section{Degradation process}

The degradation process was followed for 15 days by the observation of the color transformations of the particles placed in citrate buffer at three different $\mathrm{pHs}(2.5,3.5$ and 4.5) at room temperature (Figure 3). The color change, from the brown color of the original maghemite suspension to the yellow color typical of free iron ions, was fastest at the lowest $\mathrm{pH}$. A very similar trend was observed for both kinds of coatings. At $\mathrm{pH} 4.5$, the most similar to lysosomal conditions, complete degradation of the particles was not observed after 2 weeks.

In order to accelerate the process and also to achieve conditions more similar to the physiological medium, the degradation of the particles was then performed at $\mathrm{pH} 4.5$ and $37^{\circ} \mathrm{C}$. A significant color change from dark orange to yellow occurred within $24 \mathrm{~h}$ (Figure 4). After this period of time, particle size was significantly reduced to be $5.0 \pm 1.6$ for the CIT-MNPs $(\mathrm{p}<0.0001)$ and $4.4 \pm 2.1$ for the PAAMNPs $(\mathrm{p}<0.0001)$ (Figure 2). At the beginning of the citric

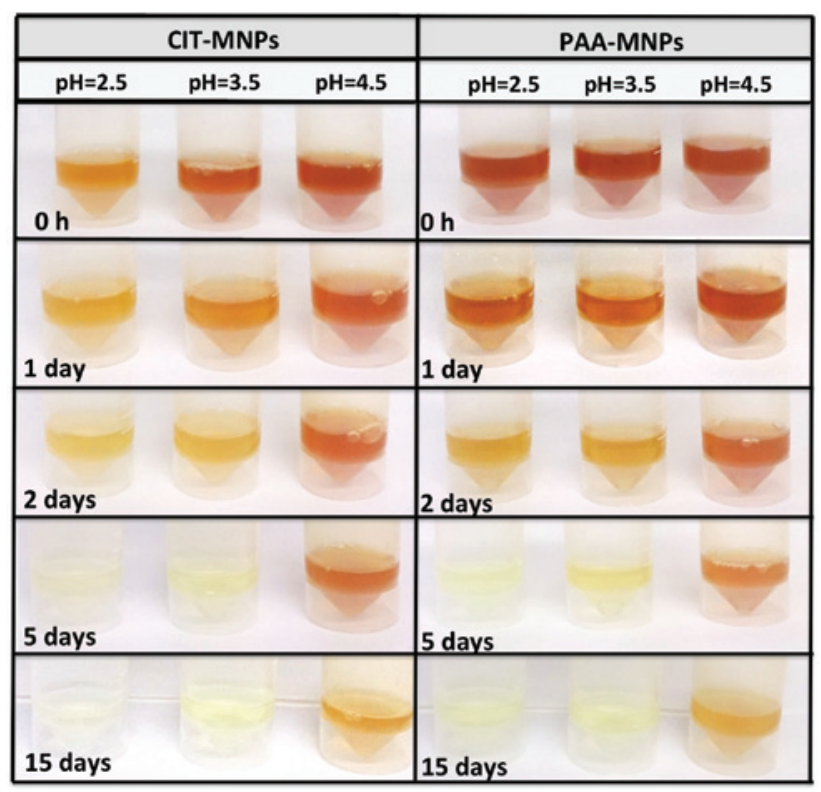

Figure 3: Degradation process at room temperature at different $\mathrm{pHs}$. Visual follow-up of the magnetic nanoparticle suspensions during the degradation process at three different pHs.

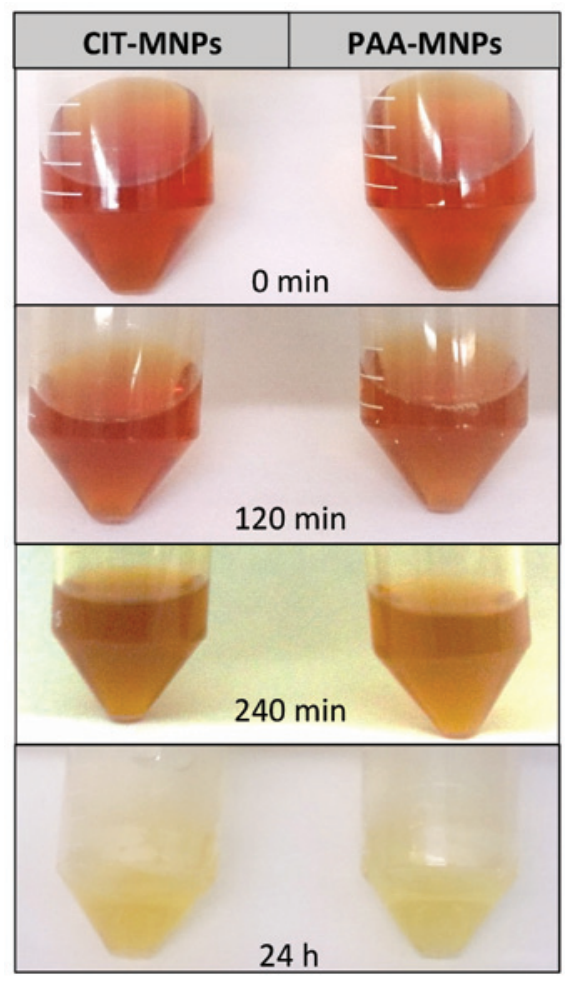

Figure 4: Degradation process in physiological conditions $\left(37^{\circ} \mathrm{C}\right.$, $\mathrm{pH}=4.5$ ).

Visual follow-up of the magnetic nanoparticle suspensions during the degradation process.

acid-coated particle degradation experiment, the size of most particles (75\%) was in the 5- to 8-nm range, whereas after $24 \mathrm{~h}, 62 \%$ of the particles presented a size in the 3to 5-nm range. A similar behavior was observed for the PAA-MNPs, where at the initial time, $64 \%$ of the particles present a size in the range between 5 and $8 \mathrm{~nm}$, while after $24 \mathrm{~h}$, most of the particles (65\%) presented sizes in the 2- to 5-nm range. The final particle size distribution after $24 \mathrm{~h}$ degradation was significantly different $(\mathrm{p}=0.001)$ between citric acid and phosphonoacetic acid coatings. It seems that PAA-MNPs degrade faster than the citric acidcoated ones.

As shown in Figure 2, particle degradation within each aggregate seems to be faster for those particles on the surface of the aggregate, meaning that the degradation process does not occur at the same speed for all particles. Therefore, degradation kinetics is probably related to the aggregation status. The degradation process was also observed through the reduction of the particle hydrodynamic size with time (Figure 5).

Field-dependent magnetization results, scaled to the magnetization values at the highest field $\left(M_{\max }\right)$, are shown in Figure 6 for the citric acid-coated magnetic 

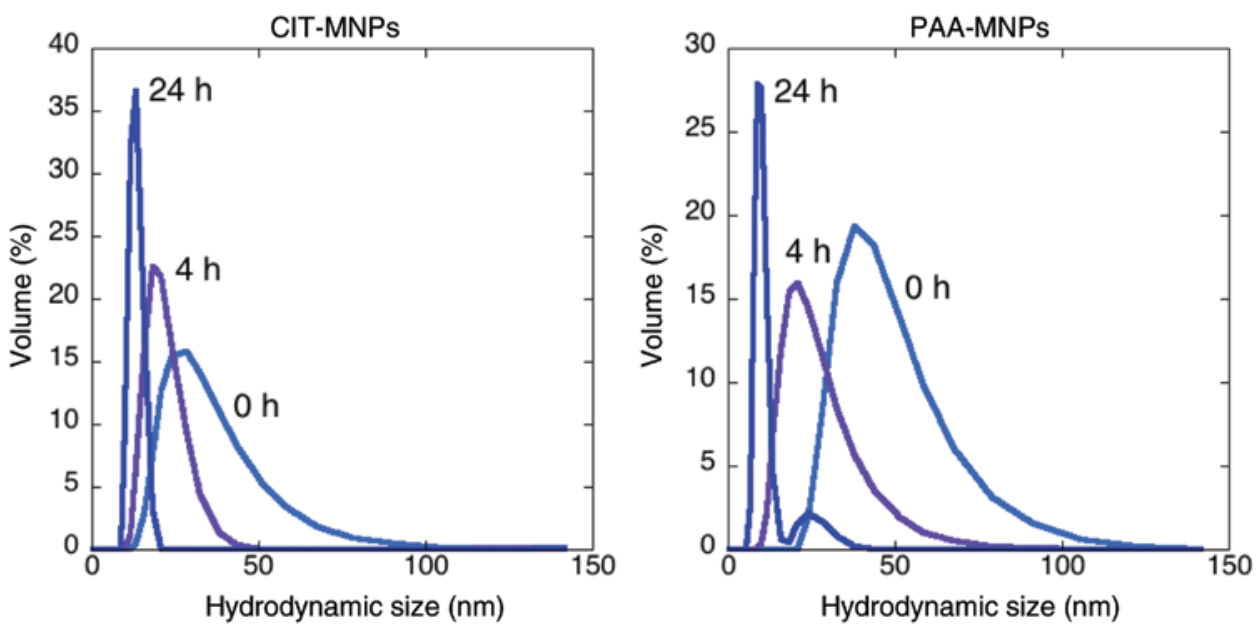

Figure 5: Hydrodynamic sizes during the degradation process $\left(37^{\circ} \mathrm{C}, \mathrm{pH}=4.5\right)$.

Hydrodynamic diameter measured by DLS at three points during the degradation process.

nanoparticles. Similar results were observed for the PAAMNPs. As expected, at $290 \mathrm{~K}$, a decrease in the saturation magnetization is observed ( $\approx$ fivefold within the 24 -h degradation process). In addition, at $5 \mathrm{~K}$, the appearance of a significant paramagnetic contribution is observed with time, probably related to the presence of iron citrate complexes. At low temperatures, the paramagnetic contribution dominates the signal in comparison with the nanoparticle contribution, and this phenomenon complicates the quantitative analysis of the particle degradation.

The temperature dependence of the $\mathrm{AC}$ magnetic susceptibility, both the in-phase $\left(\chi^{\prime}(T)\right)$ and the out-of-phase susceptibility $\left(\chi^{\prime \prime}(\mathrm{T})\right)$ components are shown in Figure 7. Above $200 \mathrm{~K}$, the out-of-phase susceptibility is zero within the accuracy of the experiments. The in-phase susceptibility shows a maximum at around $80 \mathrm{~K}$ at the beginning of the degradation experiment for both kinds of particles, accompanied by an out-of-phase susceptibility maximum at around $50 \mathrm{~K}$, related to a relaxation process.

During the degradation experiment, the temperature location of the $\chi^{\prime}$ and $\chi^{\prime \prime}$ maxima shifts toward lower temperatures reaching $35-40 \mathrm{~K}$ for the out-of-phase susceptibility component after $4 \mathrm{~h}$ under lysosomal conditions $\left(37^{\circ} \mathrm{C}\right.$ and $\left.\mathrm{pH}=4.5\right)$.

The samples prepared at the final time point, $24 \mathrm{~h}$ after the beginning of the experiment, present a different behavior. The in-phase susceptibility shows a Curie law behavior typical of paramagnetic species, indicating the degradation of the crystalline structure and the generation of other species, probably iron atoms chelated by citrates. The out-of-phase susceptibility presents a noisy maximum located at the lowest temperatures, at around $27 \mathrm{~K}$ for the citric acid-coated particles. The low signal
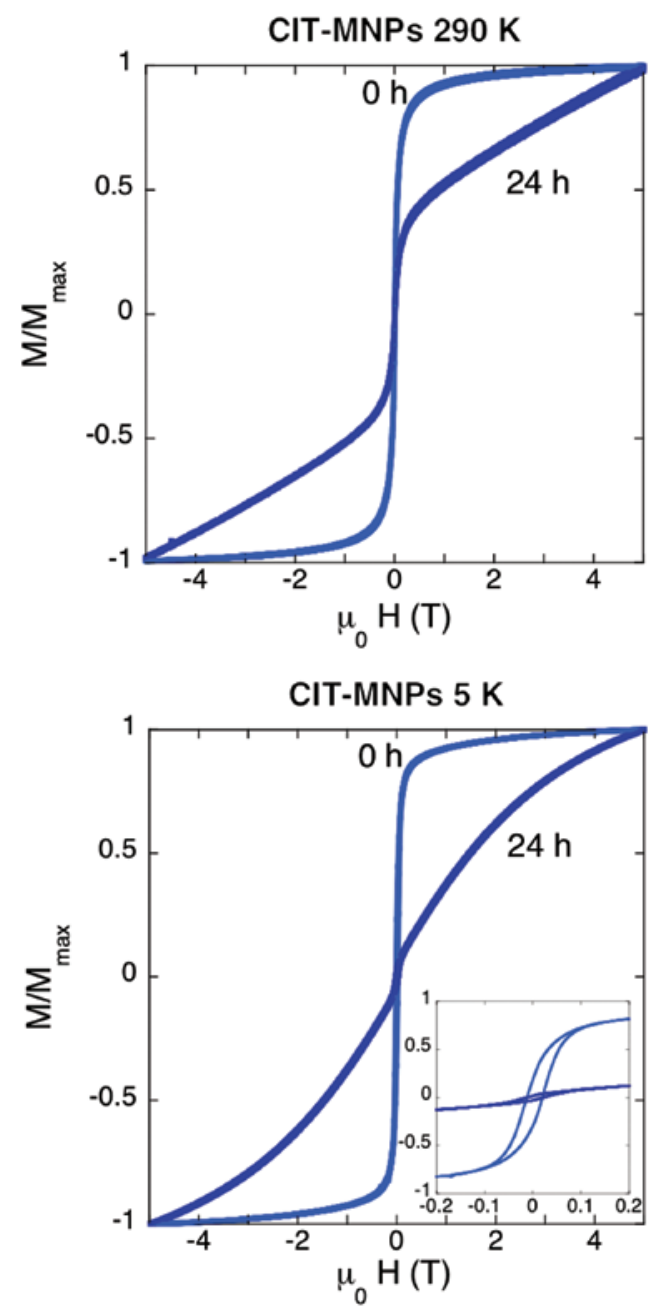

Figure 6: Field-dependent magnetization measurements during the degradation process.

Measurements have been performed at two different temperatures: $290 \mathrm{~K}$ and $5 \mathrm{~K}$. 

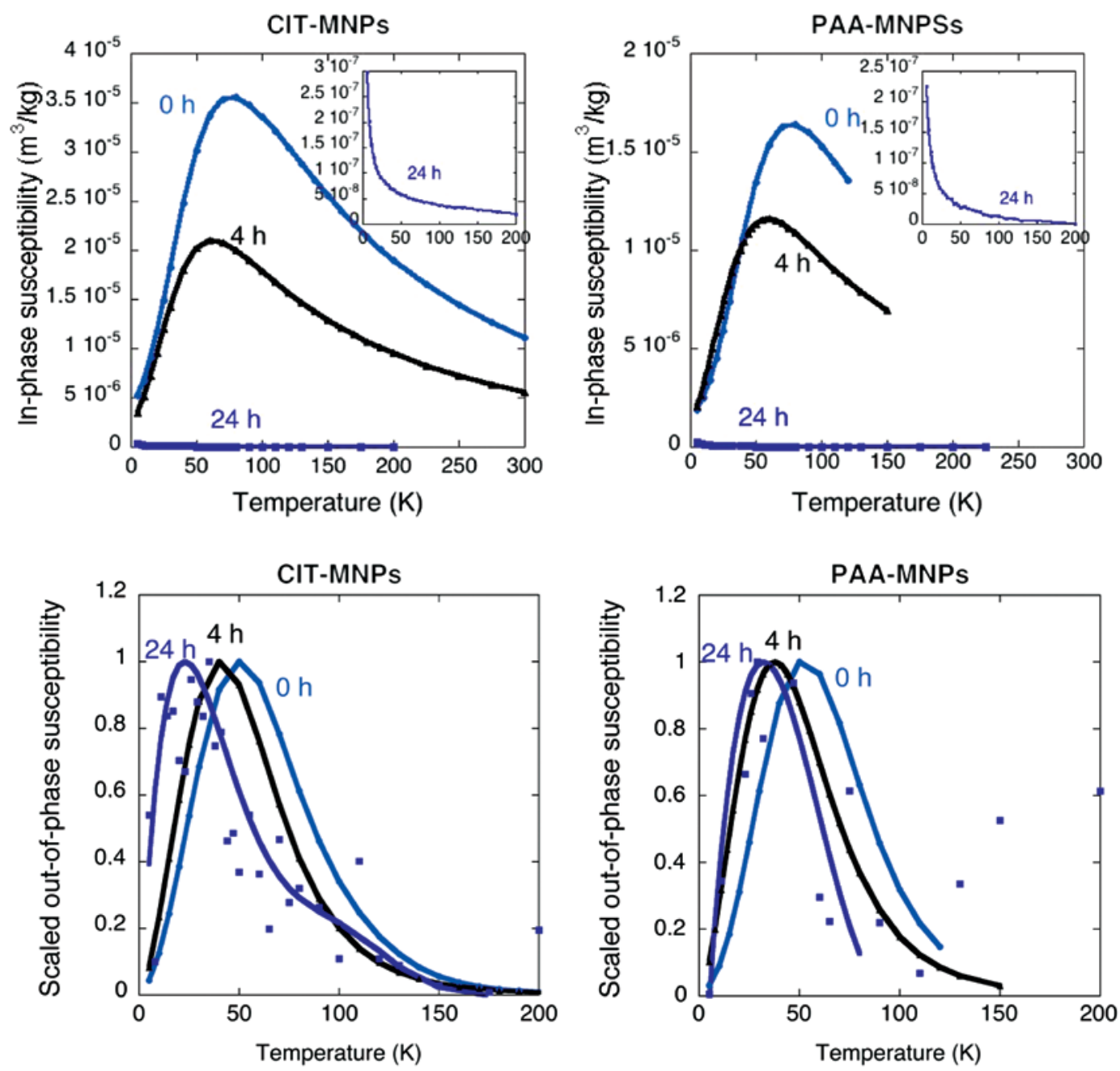

Figure 7: AC magnetic susceptibility measurements during the degradation process.

Temperature dependence of the in-phase and out-of-phase susceptibility for the two kinds of particles at three times during the degradation process $(0 \mathrm{~h}, 4 \mathrm{~h}$, and $24 \mathrm{~h}$ ). The line from the samples degraded during $24 \mathrm{~h}$ corresponds to a polynomial fit of the data points.

indicates that there is a very small fraction of the sample that is still in the form of magnetic nanoparticles.

To evaluate the importance of interparticle interactions, $\tau_{0}$ values have been calculated. This parameter is the pre-exponential factor that appears in the Arrhenius expression for the relaxation time, $\tau=\tau_{0} \exp (\mathrm{Ea} /$ $k_{\mathrm{B}} T$ ), where $k_{\mathrm{B}}$ is the Boltzmann constant and $E$ a, the single particle anisotropy energy barrier. $\tau_{0}$ values in the range between $10^{-9}$ and $10^{-12} \mathrm{~s}$ indicate negligible interparticle interactions [13]. A method to calculate $\tau_{0}$ has been described by López et al. [23], based on the complementarity of the relaxational information in $\chi^{\prime}$ and $\chi^{\prime \prime}$. Using this method, $\tau_{0}$ values have been calculated for the $0 \mathrm{~h}$ and $4 \mathrm{~h}$ time points. In samples with both coatings, $\tau_{0}$ values shift from $10^{-13} \mathrm{~s}$ at the initial time point to $10^{-11} \mathrm{~s}$ after $4 \mathrm{~h}$ of the degradation process, indicating a reduction of the interparticle interactions during the degradation process. These results are in agreement with the hydrodynamic size reduction observed by DLS (Figure 5).

\section{Discussion}

The size reduction of around $15 \%$ of the particle diameter for the citric acid-coated particles, from $6.5 \pm 1.7 \mathrm{~nm}$ to $5.6 \pm 2.4 \mathrm{~nm}$, is responsible for a temperature shift of the out-of-phase susceptibility maximum from 50 to $\approx 27 \mathrm{~K}$, reflecting the high sensitivity of these measurements to small changes in particle volume. These differences are also in agreement with the $\approx$ fivefold reduction of the saturation magnetization within the 24 -h degradation process.

A faster degradation process has been observed for PAA-MNPs in comparison with CIT-MNPs, revealing the 
importance of the particle coating on the degradation process. In our case, magnetic cores were prepared in the same batch and subsequently coated by two different procedures, allowing the study of the role of the coating on the degradation process without overlapping other effects coming from the use of different cores, as in previous works [22].

This study is a simple approximation to the degradation processes occurring in biological matrices; a more complex approach should include biological serum proteins (e.g., fetal bovine serum) in the degradation solution. It has been previously demonstrated how the formation of a protein corona in the organic media affects the hydrodynamic size of the particles and how the different particle coatings affect the formation of this protein corona [32]. Therefore, one could envisage a substantial effect of the protein corona on the degradation kinetics of the particles.

A shift of the temperature location toward lower temperatures has also been previously observed in animal models monitored during long time periods after the administration of magnetic nanoparticles [27]. In the case of animal tissues, however, it is difficult to distinguish a reduction in the particle size from a possible reduction of the dipolar interactions due to disaggregation processes $[15,23]$. Indeed, one of the challenges of using AC magnetic susceptibility measurements to evaluate the transformations suffered by magnetic nanoparticles in animal models would be to develop protocols to distinguish the processes behind the $\chi^{\prime \prime}(\mathrm{T})$ maxima shift.

When thinking of the possible degradation mechanisms, one can envisage a process where all the particles reduce their size homogeneously. However, we have found that although part of the magnetic nanoparticles suffers a significant reduction in the particle size, there is still another part that does not seem to be affected by the degradation process. In previous works, the important role played by the coating on the selection of the particles that will degrade in the first place has been described. It seems that the areas where the coating is less dense are prone to be the ones where the degradation starts [19]. Our work has also shown that particle aggregation is an additional important factor affecting the particle degradation. Thus, particles in the outer layer of the aggregate are first and severely affected by degradation while particles in the center are not affected in the first stadium of the process. This parameter strongly affects the analysis of the particles in tissue samples, as a different aggregation degree has been observed depending on the analyzed organ $[15,16]$.

For some biomedical applications, such as repeated MRI scans, slow kinetics of the magnetic nanoparticles forming the contrast agents may be desirable in a way that no additional injections are required each time an MRI image is needed. For some other uses, however, faster degradation would be desirable. Therefore, a deeper study of the parameters that affect particle degradation is of great interest.

It is fundamental to gain a better understanding on the degradation process of MNPs and the effect on their magnetic properties. In this work, we have prepared standard samples that mimic the particle degradation that will be used in the future to determine, by magnetic means, the amount of particles in tissue samples in longterm in vivo experiments.

\section{Conclusions}

The degradation of magnetic nanoparticles in a medium that mimics the lysosomal conditions has been followed by TEM, DLS, and AC magnetic susceptibility. The important role of the coating on the degradation kinetics has been verified. Also, a nonhomogenous reduction of the particle size during the degradation process has been found. The high sensitivity of the AC magnetic susceptibility measurements to particle size changes opens the way to novel approaches to evaluate the transformations of magnetic nanoparticles after systemic administration in animal models.

Acknowledgments: LG is the beneficiary of a postdoctoral grant from the AXA Research Fund and GBdS, of a sandwich-PhD grant from the Conselho Nacional de Desenvolvimento Científico e Tecnológico (CNPq - Brazil). This work was also supported by the $7^{\text {th }}$ framework programme of the European Commission Directorate-General for Research and Innovation (Nanomag 604448). X-ray diffraction, FTIR spectroscopy, and thermogravimetric and chemical analysis were carried out in the support laboratories of Instituto de Ciencia de Materiales de Madrid (CSIC). The authors would like to acknowledge the use of Servicio General de Apoyo a la Investigación-SAI, Universidad de Zaragoza.

Authors' contributions: LG supervised SR, analyzed the data, performed the magnetic measurements and wrote the manuscript. SR performed the particle degradation procedure and characterized the degradation products. GBdS and RC synthetized and characterized the magnetic nanoparticles, and co-wrote the description of the results. MDV, CMR, CJS, SV-V, and MPM discussed the results and helped in the writing of the original manuscript. 
Competing interests: The authors declare no competing interests.

\section{References}

[1] Arbab AS, Wilson LB, Ashari P, Jordan EK, Lewis BK, Frank JA. A model of lysosomal metabolism of dextran coated superparamagnetic iron oxide (SPIO) nanoparticles: implications for cellular magnetic resonance imaging. NMR Biomed 2005; 18: 383-389.

[2] Bonnecaze R, Clements M. Magnetically driven flows of suspensions of rods to deliver clot-busting drugs to dead-end arteries. Bull Am Phys Soc 2014; 59: D9.7.

[3] Bradshaw M, Clemons T, Ho D, et al. Manipulating directional cell motility using intracellular superparamagnetic nanoparticles. Nanoscale 2015; 7: 4884-4889.

[4] Calero M, Gutiérrez L, Salas G, et al. Efficient and safe internalization of magnetic iron oxide nanoparticles: two fundamental requirements for biomedical applications. Nanomed Nanotechnol Biol Med 2014; 10: 733-743.

[5] Candido RRF, Favero V, Duke M, et al. The affinity of magnetic microspheres for Schistosoma eggs. Int J Parasitol 2015; 45: 43-50.

[6] Chertok B, Cole AJ, David AE, Yang VC. Comparison of electron spin resonance spectroscopy and inductively-coupled plasma optical emission spectroscopy for biodistribution analysis of iron-oxide nanoparticles. Mol Pharm 2009; 7: 375-385.

[7] Chouly C, Pouliquen D, Lucet I, Jeune JJ, Jallet P. Development of superparamagnetic nanoparticles for MRI: effect of particle size, charge and surface nature on biodistribution. J Microencapsulation 1996; 13: 245-255.

[8] Colombo M, Carregal-Romero S, Casula MF, et al. Biological applications of magnetic nanoparticles. Chem Soc Rev 2012; 41:4306-4334.

[9] Costo R, Bello V, Robic C, et al. Ultrasmall iron oxide nanoparticles for biomedical applications: improving the colloidal and magnetic properties. Langmuir 2011; 28: 178-185.

[10] Costo R, Morales MP, Veintemillas-Verdaguer S. Improving magnetic properties of ultrasmall magnetic nanoparticles by biocompatible coatings. J Appl Phys 2015; 117: 064311.

[11] Dobson J. Magnetic nanoparticles for drug delivery. Drug Dev Res 2006; 67: 55-60.

[12] Gamarra LF. Ferromagnetic resonance for the quantification of superparamagnetic iron oxide nanoparticles in biological materials. Int J Nanomed 2010; 5: 203.

[13] Garcia-Palacios J. On the statics and dynamics of magnetoanisotropic nanoparticles. Adv Chem Phys 2000; 112: 1-210.

[14] Gutierrez L, Costo R, Gruttner C, et al. Synthesis methods to prepare single- and multi-core iron oxide nanoparticles for biomedical applications. Dalton Trans 2015; 44: 2943-2952.

[15] Gutiérrez L, Mejías R, Barber DF, et al. Ac magnetic susceptibility study of in vivo nanoparticle biodistribution. J Phys D: Appl Phys 2011; 44: 255002.

[16] Gutierrez L, Mejias R, Lazaro FJ, Serna CJ, Barber DF, Morales MP. Effect of anesthesia on magnetic nanoparticle biodistribution after intravenous injection. IEEE Trans Magn 2013; 49: 398-401.

[17] Gutierrez L, Morales MP, Lazaro FJ. Prospects for magnetic nanoparticles in systemic administration: synthesis and quantitative detection. Phys Chem Chem Phys 2014; 16: 4456-4464.

[18] Hergt R, Dutz S, Müller R, Zeisberger M. Magnetic particle hyperthermia: nanoparticle magnetism and materials development for cancer therapy. J Phys: Condens Matter 2006; 18: S2919.

[19] Lartigue L, Alloyeau D, Kolosnjaj-Tabi J, et al. Biodegradation of iron oxide nanocubes: high-resolution in situ monitoring. ACS Nano 2013; 7: 3939-3952.

[20] Lee J, Lilly GD, Doty RC, Podsiadlo P, Kotov NA. In vitro toxicity testing of nanoparticles in 3D cell culture. Small 2009; 5: 1213-1221.

[21] Lesieur S, Grabielle-Madelmont C, Ménager C, et al. Evidence of surfactant-induced formation of transient pores in lipid bilayers by using magnetic-fluid-loaded liposomes. J Am Chem Soc 2003; 125: 5266-5267.

[22] Lévy M, Lagarde F, Maraloiu V-A, et al. Degradability of superparamagnetic nanoparticles in a model of intracellular environment: follow-up of magnetic, structural and chemical properties. Nanotechnology 2010; 21: 395103.

[23] López A, Gutiérrez L, Lázaro FJ. The role of dipolar interaction in the quantitative determination of particulate magnetic carriers in biological tissues. Phys Med Biol 2007; 52: 5043-5056.

[24] Martina M-S, Fortin J-P, Ménager C, et al. Generation of superparamagnetic liposomes revealed as highly efficient MRI contrast agents for in vivo imaging. J Am Chem Soc 2005; 127 : 10676-10685.

[25] Massart R. Preparation of aqueous magnetic liquids in alkaline and acidic media. IEEE Trans Magnetics 1981; 17: 1247-1248.

[26] Meddahi-Pellé A, Legrand A, Marcellan A, Louedec L, Letourneur D, Leibler L. Organ repair, hemostasis, and in vivo bonding of medical devices by aqueous solutions of nanoparticles. Angew Chem Int Ed 2014; 53: 6369-6373.

[27] Mejías R, Gutiérrez L, Salas G, et al. Long term biotransformation and toxicity of dimercaptosuccinic acid-coated magnetic nanoparticles support their use in biomedical applications. J Controlled Release 2013; 171: 225-233.

[28] Ruiz A, Hernández Y, Cabal C, et al. Biodistribution and pharmacokinetics of uniform magnetite nanoparticles chemically modified with polyethylene glycol. Nanoscale 2013; 5: 11400-11408.

[29] Sasaki T, Iwasaki N, Kohno K, et al. Magnetic nanoparticles for improving cell invasion in tissue engineering. J Biomed Mater Res A 2008; 86A: 969-978.

[30] Shinkai M, Le B, Honda H, et al. Targeting hyperthermia for renal cell carcinoma using human $\mathrm{MN}$ antigen-specific magnetoliposomes. Jpn J Cancer Res 2001; 92: 1138-1146.

[31] Sun C, Lee JSH, Zhang M. Magnetic nanoparticles in MR imaging and drug delivery. Adv Drug Deliv Rev 2008; 60: 1252-1265.

[32] Wiogo HTR, Lim M, Bulmus V, Gutiérrez L, Woodward RC, Amal R. Insight into serum protein interactions with functionalized magnetic nanoparticles in biological media. Langmuir 2012; 28: 4346-4356. 